\title{
Beriberi, a Severe Complication after Metabolic Surgery - Review of the Literature
}

\author{
Christine Stroh $^{\mathrm{a}} \quad$ Frank Meyer $^{\mathrm{b}}$ Thomas Manger $^{\mathrm{a}}$ \\ aSRH Municipal Hospital, Department of General, Abdominal and Pediatric Surgery, Gera, \\ Germany; ${ }^{b}$ University Hospital, Department of General, Abdominal and Vascular Surgery, \\ Magdeburg, Germany
}

\section{Key Words}

Bariatric surgery · Lack of thiamine · Bariatric Beriberi · Wernicke's encephalopathy ·

Wernicke-Korsakoff syndrome

\begin{abstract}
Background: The epidemic growth of morbid obesity has led to an increase in the number of bariatric interventions. During the distribution process of bariatric surgical interventions, the risk for severe nutritious complications such as bariatric beriberi can rise. Methods: By means of systematic literature review, epidemiological data, clinical characteristics and diagnostic as well as therapeutic recommendations for bariatric beriberi were elicited. Databases and registries such as PubMed, Cochrane and Ovid were searched for a defined time period with the key words 'lack of thiamine' / 'Wernicke-Korsakoff syndrome' / 'encephalopathy' after bariatric surgical interventions. Results: Up to December 2013, overall 255 patients had been found as published cases, indicating that the risk for the postoperative occurrence of thiamine deficiency and Wernicke-Korsakoff syndrome is increased in women. In addition, the risk correlates with patient's age. The majority of patients developed symptoms of a dry beriberi with peripheral neuritis, ataxia and paraplegia, indicating an advanced stage of disease approximately 4-12 weeks postoperatively. Laboratory analysis in case of a suspicious clinical finding is the appropriate diagnostics. As treatment, prompt initiation of parenteral thiamine substitution under clinical monitoring is required. Conclusion: Bariatric beriberi can occur within the first 1-3 postoperative months. To minimize the risk of severe consequences, immediate substitution of thiamine in clinical suspicion or prolonged parenteral nutrition is necessary. A delayed diagnosis or missing the correct diagnosis can lead to irreversible damages of the CNS with coma and fatal outcome. Knowledge on the subject, including development of thiamine deficiency, symptomatology and emergency treatment, are considered essential for bariatric surgeons but also for further medical disciplines involved in treatment.




\section{Introduction}

Obesity is one of the greatest challenges to health in the 21 century. According to data of the International Association for the Study of Obesity (IASO), Germany ranks first in prevalence of obesity for both genders [1]. The Federal Office of Statistics revealed that in 2009 $51.4 \%$ of the population was overweight or obese. The average BMI of the German population in 2009 was $25.7 \mathrm{~kg} / \mathrm{m}^{2}$, with $60.1 \%$ of men and $42.9 \%$ of women overweight [2]. Life expectancy is markedly shortened by obesity, particularly in young obese individuals. The mortality risk rises between 6- and 12-fold of that of the normal population, with a 12-year and a 9-year reduction in life expectancy for overweight men and women, respectively. For grade 3 obesity, a 20-year statistically corroborated reduction in life expectancy has been identified [3].

The Swedish obesity study has shown the long-term effect of obesity surgery with regard to weight loss and amelioration of comorbidities [4].

In Germany, the number of metabolic and bariatric procedures has been increased during the last years with currently more than 5,000 procedures annually. Especially the number of Roux-en-Y-gastric bypass (RYGB) operations has increased. Scopinaro procedures such as biliopancreatic diversions without (BPD) or with duodenal switch (BPD/DS) were only performed in few hospitals. Standardized follow-up, including laboratory tests, is important to avoid complications like vitamin and mineral deficiencies or malnutrition. It is therefore problematic that in Germany the follow-up examinations after obesity surgery are not covered by the health insurance systems. Moreover, the knowledge of most physicians regarding side effects and nutrient complications after several procedures is low.

The aim of this short overview was to summarize the incidence, symptomatology, and appropriate management of beriberi post bariatric surgery based on a case in our hospital (severe beriberi after BPD because of insufficient weight loss after former gastric banding) and selected data from the literature.

\section{Methods}

By means of systematic literature review, epidemiological data, clinical characteristics, and diagnostics as well as therapeutic recommendations for bariatric beriberi were elicited using data from databases such as 'PubMed', 'Cochrane', and 'Ovid' were searched for a defined time period (key words, 'lack of thiamine', 'Wernicke Korsakoff syndrome', 'encephalopathy' after bariatric surgical interventions).

\section{Results}

A review of representative data from the literature indicates that the risk to develop beriberi is higher for female than for male patients. More than $90 \%$ of reported cases of beriberi post bariatric surgery were observed in female patients [5]. The risk of developing beriberi is strongly correlated to the patient's age. Own data analysis revealed a mean patients' age of 34.1 (range 14-55) years. According to published literature, patients with beriberi were between 23 and 55 years old [5]. Most patients developed beriberi within a time frame of 4 to 12 weeks after surgery.

After BPD, the incidence of beriberi is reported with $0.18 \%$ [6]. A questionnaire focusing on neurological complications after bariatric surgery resulted in an incidence of 5.9 cases in 10,000 operations. The problem of this investigation is that only $31.8 \%$ of the patients answered the questionnaires. The incidence of vitamin B1 deficiency was 1.7 cases in 10,000 bariatric procedures [7]. 
Stroh et al.: Beriberi, a Severe Complication after Metabolic Surgery - Review of the Literature

Table 1. List of relevant recent publications on beriberi

\begin{tabular}{lllll}
\hline Author & $\begin{array}{l}\text { Number of } \\
\text { patients }\end{array}$ & Gender & Age, years & $\begin{array}{l}\text { Surgical } \\
\text { procedure }\end{array}$ \\
\hline Singh and Kumar, 2007 [5] & 32 & $\begin{array}{l}27 \text { females } \\
1 \text { male }\end{array}$ & \\
$\begin{array}{l}\text { Matrana et al., 2009 [21] } \\
\text { Stroh et al., 2010 [22] }\end{array}$ & 1 & $\begin{array}{l}197 \text { females } \\
\text { female }\end{array}$ & 34.5 & 27 \\
Ianelli et al., 2010 [23] & 118 & RYGB & RYGB \\
Walker and Kepner, 2012 [24] & 1 & $\begin{array}{l}1 \text { female } \\
1 \text { male }\end{array}$ & 25 & LAGB \\
Becker et al., 2012 [25] & 1 & 49 & SG \\
\hline Goselink et al., 2012 [26] & 1 & & & \\
\hline
\end{tabular}

A certain number of morbidly obese patients already suffer from vitamin, mineral and calcium metabolism disorders preoperatively. Therefore, preoperative diagnosis, prophylaxis, and therapy of these specific deficiencies are necessary to avoid life-threatening complications [8].

Summarizing these results, the following recommendations can be derived:

- preoperative diagnosis of vitamin deficiencies,

- appropriate compensation of preoperative deficiencies in trace elements and calcium,

- multivitamin supplementation in case of long-term postoperative vomiting,

- instant diagnosis of unexplained symptoms (vision, numbness, cramps, burning feet),

- targeted compensation of detected deficiencies,

- life-long supplementation and controlled adjustment,

- periodic follow-up examinations to close inspection intervals.

Not only bariatric surgeons but also all other physicians involved in the medical aftercare of such patients should be aware of the risks of vitamin, trace element and electrolyte deficiencies after bariatric surgery and how these deficiencies can be diagnosed and treated $[9,10]$. In case of clinical suspicion or deferred parenteral nutrition, the immediate introduction of a thiamine substitution is necessary to minimize the risk of catastrophic consequences. A delayed diagnosis or misdiagnosis of beriberi can lead to irreversible damage of the CNS with coma and even fatal outcome. Timely diagnosis and initiation of treatment are possible only in close interdisciplinary cooperation in centers or network structures of bariatric surgery including medical specialists and family practitioners in outpatient clinics.

\section{Discussion}

\section{Pathophysiology}

Thiamine is a water-soluble vitamin resorbed in the proximal jejunum with support of an active transport system. Thiamine reserves in the body amounted to only $30 \mathrm{mg}$ and are depleted as quickly as 20 days of inadequate oral intake [11,12]. Reasons for vitamin B1 deficiency are persistent vomiting, food with low vitamin B1 content, or an excessive need of vitamin B.

Resorbed thiamine is available in the body in a non-phosphorylated or a phosphorylated form (thiamine mono-, pyro- and triphosphate). Thiamine pyrophosphate is the coenzyme of several enzymatic reactions of cell regulatory processes, biosynthesis, and metabolism [11]. Thiamine pyrophosphate is a cofactor of transketolase and a catalyst of the pentose phosphate cycles and their product NADPH. Thiamine deficiency therefore results 
in defects of cell synthesis, repair, and replication. In the CNS, thiamine deficiency causes defects of acetylcholine synthesis. In addition, a sufficient amount of thiamine pyrophosphate in nerve and muscle is important for the activation of the transport of sodium and potassium. Therefore, it is not surprising that deficiency of thiamine pyrophosphate causes reduced sensomotoric activity, muscle atrophy, and neuropathy. Furthermore, thiamine is important for:

- carbohydrate metabolism,

- citrate cycles,

- synthesis of nucleic acids and nucleotides,

- transport of ions for sensomotoric activity and nervous function,

- synthesis of neurotransmitters and cognitive functions.

Therefore, thiamine deficiency can result in various partially life-threatening complications.

\section{Incidence}

Several studies have found a beriberi incidence prior to bariatric surgery procedures of approximately $15.5 \%$ [12-14]. In women, the thiamine level is lower than in men [9]. A retrospective analysis from the literature indicated that $29 \%$ of patients suffer from thiamine deficiency prior to bariatric surgery [15]. Reasons were not detected and reported. However, the preoperative thiamine level should be determined, and in case of deficiency supplementation has to be initiated prior to surgical intervention.

\section{Postoperative Deficiency}

After bariatric surgery a latent preoperative thiamine deficiency can become symptomatic and manifest.

Restrictive procedures can cause thiamine deficiency due to:

- low intake of nutrition and vitamins,

- postoperative prolonged nutrition with fluids,

- recurrent postoperative emesis [16-19].

Malabsorptive procedures in bariatric surgery can lead to thiamine deficiency due to:

- low intake of nutrition,

- reduction or loss of appetite,

- reduced thiamine resorption due to a deficiency of gastric acid,

- duodenal exclusion,

- exclusion of the proximal jejunum,

- changes of vitamin resorption at the common channel with a length of 50 to $100 \mathrm{~cm}$,

- hyperemesis.

Most patients develop thiamine deficiency within 1-3 months postoperatively caused by hyperemesis. In patients with long-term parenteral nutrition, vitamin B1 supplementation is required.

\section{Symptoms}

Wet Beriberi

Wet beriberi is characterized by cardiac symptoms, cardiac insufficiency with tachycardia, right heart insufficiency and respiratory symptoms, vasodilatation edema, and hypertension.

Dry Beriberi

Dry beriberi is more frequent than wet beriberi. Symptoms are neuritis, neuropathy, especially at the lower extremities, and muscle pain with atrophy and paraplegia. 
Stroh et al.: Beriberi, a Severe Complication after Metabolic Surgery - Review of the Literature

Cerebral Beriberi

Cerebral beriberi is a special form of dry beriberi associated with an acute severe thiamine deficiency. This influences the CNS and the spinal cord and results in Wernicke-Korsakoff syndrome. Ataxia and oculomotor problems are further symptoms. In severe stages, coma is possible. If treated early the prognosis of beriberi is good prognosis but mortality still ranges between 10 and 20\%. Despite early initiation of treatment, complete remission is rare as cognitive changes are not reversible in most cases [20].

\section{Diagnostics}

Thiamine deficiency can easily be diagnosed by determining blood thiamine levels; however, clinical symptoms need to be recognized and appropriately interpreted.

\section{Prophylaxis and Therapy}

Thiamine supplementation of $1.1 \mathrm{mg} /$ day for women and of $1.2-1.5 \mathrm{mg} /$ day for men is necessary to avoid deficiency. In early stage of neuropathy, 20-30 mg/day are necessary for a complete convalescence of the patient. If symptoms of Wernicke's encephalopathy are diagnosed, $100 \mathrm{mg} /$ day of thiamine intravenously over a time period of several days are required followed by an enteral supplementation with high doses $[16,17]$. During the treatment of vitamin B1 deficiency, an additional supplementation with all other vitamins of the B group is important. Therefore, bariatric surgery patients should be basically treated with vitamin $B$ supplements to avoid complications.

\section{Recommended Follow-Up Algorithm after Malabsorptive Procedures}

The patient and the general practitioner should be informed about the principles of the procedure (including a schematic diagram) to be applied and a description of several severe complications. Moreover, it is of utmost importance to provide both the patient in the physician with an emergency call number of the operating center in order to get help in case of complications or contingencies.

An extensive laboratory examination to evaluate the nutritional status is a prerequisite for all malabsorptive procedures, including tests on fasting glucose levels, liver function, renal function, bone alkaline phosphatase, albumin, prealbumin, prothrombin and INR, vitamin B1, folates, vitamin B12, vitamin D3, iron and ferritin, parathormone, vitamin E, vitamin A as well as hemoglobin and electrolytes [27]. If this examination revealed any nutritional deficiency, a preoperative supplementation should be started.

In the postoperative course, these parameters should be evaluated after 1, 3, 6 and 12 months after surgery and later on twice a year to adapt supplementation and to avoid severe complications. Parenteral supplementation should be given in case of vitamin D3, severe iron, vitamin B1 and micronutrient deficiency.

Long-life supplementation of vitamins and micronutrients should be aligned to the following dietary recommendations:

- protein: 50-60 g/day

- ferrum: 100-160 mg/day

- vitamin B12: 1,000 $\mathrm{\mu g} /$ month

- folate: $400 \mathrm{mg} /$ day

- calcium: 2,000 mg/day

- vitamin D: $400 \mathrm{UI} /$ day

- vitamin E: multivitamins

- vitamin K: no evaluted data [10]. 
Stroh et al.: Beriberi, a Severe Complication after Metabolic Surgery - Review of the Literature

\section{Conclusion}

Published data indicates that the risk for the postoperative occurrence of thiamine deficiency and Wernicke-Korsakoff syndrome is distinctly increased in women. In addition, the risk correlates with patient's age. The majority of patients developed symptoms of a dry beriberi 4-12 weeks postoperatively, with peripheral neuritis, ataxia and paraplegia indicating an advanced stage of disease approximately.

Laboratory analysis in case of suspicious clinical findings is the appropriate diagnostics. If thiamine deficiency is found, prompt initiation of parenteral thiamine substitution under clinical monitoring is required. To minimize the risk of severe consequences, immediate thiamine substitution or prolonged parenteral nutrition is necessary in clinically suspicious patients. A delayed diagnosis or missing the correct diagnosis can lead to irreversible damages of the CNS with coma and fatal outcome. Knowledge on the subject, including development of thiamine deficiency, symptomatology and emergency treatment, can be considered essential for bariatric surgeons but also for other physicians involved in treatment after bariatric surgery [9].

\section{Acknowledgement}

The German Nationwide survey on bariatric surgery is supported by Ministry of Research and Education Germany (BMBF) grant number 01GI1124. The responsible investigator is Christine Stroh.

\section{Disclosure Statement}

There is no conflict of interest.

Christine Stroh, Frank Meyer and Thomas Manger confirm that there are no links to the firm whose products are mentioned in the article or to a firm marketing a competing product. The topic is presented in an independent light, and the information outlined is product neutral.

\section{References}

1 Lampert T: Übergewicht und Adipositas in Deutschland. RKI Epidemiol Bull 2007;18:155-156.

2 Statistisches-Bundesamt: Mikrozensus - Fragen zur Gesundheit - Körpermaße der Bevölkerung 2009. Wiesbaden, Statistisches Bundesamt 2010.

-3 Fontaine KR, Redden DT, Wang C, Westfall AO, Allison DB: Years of life lost due to obesity. JAMA 2003;289: 187-193.

-4 Sjöström L, Narbro K, Sjöström CD, Karason K, Larsson B, Wedel H, Lystig T, Sullivan M, Bouchard C, Bengtsson B, Dahlgren S, Gummesson A, Jacobsen P, Karlsson J, Lindross AK, Lönroth H, Naslund I, Olbers T, Stenlof K, Torgerson J, Agren J, Carlsson L: Effects of bariatric surgery on mortality in Swedish obese subjects. N Engl J Med 2007;357:741-752.

5 Singh S, Kumar A: Wernicke encephalopathy after obesity surgery. A systematic review. Neurology 2007;68: 807-811.

6 Primavera A, Brusa G, Novello P, etal: Wernicke-Korsakoff encephalopathy following biliopancreatic diversion. Obes Surg 1993;3:175-177.

-7 Chang CG, Adams-Huet B, Provost DA: Acute post-gastric reduction surgery (APGARS) neuropathy. Obes Surg 2004;14:182-189.

8 Flancbaum L, Belsley S, Drake V, Colarusso T, Tayler E: Preoperative nutritional status of patients undergoing Roux-en-Y gastric bypass for morbid obesity. J Gastrointest Surg 2006;10:1033-1037.

-9 Stroh C, Meyer F, Manger T: Increased requirements for the challenging abdominosurgical management of morbid obesity - what does the abdominal surgeon need to know? (In German). Zentralbl Chir 2013;138: 456-462.

10 Stroh C, Benedix F, Meyer F, Manger T: Nutrient deficiencies after bariatric surgery - systematic literature review and suggestions for diagnostics and treatment (in German). Zentralbl Chir 2013; DOI: $10.1055 / \mathrm{s}-0032-1328594$. 
Stroh et al.: Beriberi, a Severe Complication after Metabolic Surgery - Review of the Literature

11 Sechi GP, Serra A: Wernicke's encephalopathy: new clinical settings and recent advances in diagnosis and management. Lancet Neurol 2007;6:442-455.

12 Antozzi P, Lester C, Soto F, et al: Thiamin deficiency in an obese population undergoing laparoscopic surgery. Surg Obes Relat Dis 2005;1:264-265.

13 Breiter A, Marema RT, Buffington CK: Addressing preoperative nutrient deficiencies. Surg Obes Relat Dis 2005; $1: 285$.

$\checkmark 14$ Carrodeguas L, Kaidar-Person 0, Szomstein S, Antozzi P, Rosenthal R. Preoperative thiamine deficiency in obese population undergoing laparoscopic bariatric surgery. Surg Obes Relat Dis 2005;1:517-522; discussion 522.

15 Al-Fahad T, Ismal A, Soliman MO, Khoursheed M: Very early onset of Wernicke's encephalopathy after gastric bypass. Obes Surg 2006;16:671-672.

16 Cirignotta F, Manconi M, Mondini S, Buzzi G, Ambrosetto P: Wernicke-Korsakoff encephalopathy and polyneuropathy after gastroplasty for morbid obesity: report of a case. Arch Neurol 2000;57:1356-1359.

17 Fandino N, Benchimol AK, Fandiño LN, Barroso FL, Coutinho WF, Appolinário JC: Eating avoidance disorder and Wernicke-Korsakoff syndrome following gastric bypass: an under-diagnosed association. Obes Surg 2005;15:1207-1210.

18 Madan AK, Orth WS, Tichnasky DS, Ternovits CA: Vitamin and trace mineral levels after laparoscopic gastric bypass. Obes Surg 2006;16:603-606.

19 Salas-Salvado J, Garcia-Lorda P, Cuatrecasas G, et al: Wernicke’s syndrome after bariatric surgery. Clin Nutr 2000;19:371-373.

20 Kramer LD, Locke GE: Wernicke’s encephalopathy. Complication of gastric plication. J Clin Gastroenterol 1987; 9:549-552.

21 Matrana MR, Vasireddy S, Davis WE: The skinny on a growing problem: dry beriberi after bariatric surgery. Ann Intern Med 2008;149:842-844.

-22 Stroh C, Meyer F, Lippert H, Manger T: Die Beriberi eine seltene, aber schwerwiegende Komplikation nach bariatrischen Eingriffen: Inzidenz, Symptomatologie, Therapie und Prävention. Viszeralmedizin 2010;26: 41-46.

23 Ianelli A, Addeo P, Novellas S, Gugenheim J: Wernicke’s encephalopathy after laparoscopic Roux-en-Y gastric bypass: a misdiagnosed complication. Obes Surg 2010;20:1594-1596.

24 Walker J, Kepner A: Wernicke's encephalopathy presenting as acute psychosis after gastric bypass. J Emerg Med 2012;43:811-814.

25 Becker DA, Ingala EE, Martinez-Lage M, Price RS, Galetta SL: Dry beriberi and Wernicke's encephalopathy following gastric lap band surgery. J Clin Neurosci 2012;19:1050-1052.

26 Goselink RJ, Harlaar JJ, Vermeij FH, Biter UL, Mannaerts GH, den Heijer T: Beriberi after bariatric surgery. Ned Tijdschr Geneeskd 2012;155:A4500.

27 Fried M, Yumuk V, Oppert JM, Scopinaro N, Torres A, Weiner R, Yashkov Y, Frühbeck G: Interdisciplinary European guidelines on metabolic and bariatric surgery. Obes Surg 2014;24:42-55. 